\title{
The importance of constraint relief caused by rubber cavitation in the toughening of epoxy
}

\author{
A. F. YEE, DONGMING LI, XIAOWEI LI \\ Department of Materials Science and Engineering, University of Michigan, Ann Arbor, \\ MI 48109, USA
}

\begin{abstract}
Many rubber-toughened epoxies are thought to derive the bulk of their toughness through the processes of rubber cavitation and plastic shear-yielding in the epoxy matrix. Constraint relief has been considered to be a key mechanism which allows extra plastic shear deformation to occur. The present work attempts to provide direct experimental evidence of the constraint relief effect by combining testing geometries that vary the degree of constraint with microscopic observations. The results show that the success of a rubber as a toughening agent for epoxies is closely related to its ability to cavitate. Evidence for local constraint relief is presented. Upon cavitation of the rubber, the stress state in a specimen with initial constraint is found to change to a plane stress state. The constraint relief circumvents or delays the crack initiation in the matrix, which allows more plastic deformation to occur.
\end{abstract}

\section{Introduction}

The use of a carboxyl-terminated butadiene-acrylonitrile (CTBN) rubber to toughen epoxy resins can be very effective, sometimes effecting a ten-fold or more increase in GIC. For this reason, the toughening mechanisms of CTBN rubber-modified epoxies have long been the research interest of many researchers, and the mechanisms proposed so far are reviewed by Garg and Mai [1]. The most important mechanism is the cavitation mechanism, which attributes the fracture toughness increase to cavitation of CTBN rubber particles and the induced dilatational deformation and shear yielding in the surrounding epoxy matrix. This mechanism was first proposed by Bascom and Hunston $[2,3]$ based on the SEM examination of fracture surfaces of CTBN rubber-modified epoxy specimens. In this first version of the cavitation mechanism, the toughness increase is attributed to the increase in the critical deformation zone due to CTBN rubber addition, but the role of CTBN rubber cavitation was not explained. Later, this mechanism was further developed by Yee and Pearson $[4,5]$ and Kinloch et al. $[6,7]$ based on mechanical and microscopy studies. Yee and Pearson $[4,5]$ suggested that the deformation processes are: (1) localized cavitation in the rubber, (2) plastic shear yielding in the epoxy matrix. They further hypothesized that the relief of triaxial tensile constraint by rubber cavitation is a very important step in toughening. However, this plausible argument remains to be experimentally verified.

The purpose of this work was to provide direct experimental evidence of this constraint relief effect. Multi-axial tensile stress states are created by the following two experiments: (1) the symmetrical double-edge double-notched four-point bend
(SDEDN 4-PB) test, and (2) the symmetrical doublegrooved strip tensile test. Combining these two experiments with various microscopy techniques, we have examined the fracture mechanism of pure epoxy under multiaxial tensile stress states, and the effect of rubber cavitation on the deformation and fracture of modified epoxy materials. In a following paper, we will evaluate the contribution of the dilatation process to the total energy absorption by an energy approach.

\section{Experimental procedure}

\subsection{Materials}

The materials used in the present research consist of Epoxy DER ${ }^{\circledR} 331$, HYCAR ${ }^{\circledR}$ CTB $2000 \times 162$ rubber and $\mathrm{HYCAR}^{\circledR}$ CTBN $1300 \times 8$ rubber. Epoxy DER 331 is a liquid diglycidyl ether of bisphenol-A (DGEBA) produced by the Dow Chemical Co. The curing agent is piperidine, which was used as-received from Aldrich Chemical Co. The CTB $2000 \times 162$ is a carboxyl-terminated butadiene homopolymer, and CTBN $1300 \times 8$ rubber is a carboxyl-terminated acrylonitrile-butadiene copolymer. They are produced by the B.F. Goodrich Co. Details about these two rubbers can be found elsewhere [8].

The CTB rubber-toughened epoxy consisted of 100 parts of DER 331, 2.5 parts of HYCAR CTB 2000 $\times 162$ and 5 part of piperidine. The CTBN-rubber toughened epoxies consisted of 100 parts of DER 331, 5 parts of piperidine, 2.5, 5, 7.5, 10 and 13.5 parts of HYCAR CTBN $1300 \times 8$. The preparation of these rubber-modified epoxies is similar to that described by Yee and Pearson [4]: They are cured at $120^{\circ} \mathrm{C}$ for $16 \mathrm{~h}$ in a Teflon ${ }^{\circledR}$-coated mould. The mechanical properties of these materials are listed in Table I. 


\begin{tabular}{llll}
\hline Materials & $\begin{array}{l}E \\
(\mathrm{GPa})\end{array}$ & $\begin{array}{l}\sigma_{\mathrm{Y}} \\
(\mathrm{MPa})\end{array}$ & $\begin{array}{l}K_{\mathrm{IC}} \\
\left(\mathrm{MPa} \mathrm{m} \mathbf{m}^{1 / 2}\right)\end{array}$ \\
\hline Epoxy: DER 331 & 2.94 & 74 & 0.85 \\
Epoxy/CTBN: DER 331/pip/CTBN-8 7.5 p.h.r. & 2.7 & 68 & 1.9 \\
Epoxy/CTBN: DER 331/pip/CTBN-8 10 p.h.r. & 2.6 & 63 & 2.1 \\
Epoxy/CTBN: DER 331/pip/CTB 2.5 p.h.r. & 2.83 & 71 & 1.05 \\
\hline
\end{tabular}

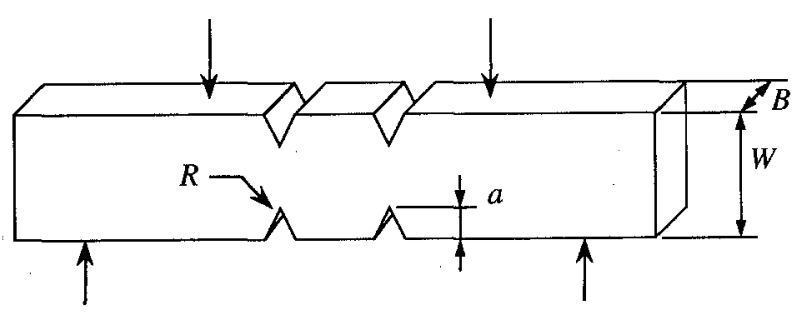

Figure 1 Illustration of a symmetrical double-edge double-notched (SDEDN) specimen, where $W=19.1 \mathrm{~mm}, B=6.3 \mathrm{~mm}, a / W=0.25$, and $R=0.5$ or $1.5 \mathrm{~mm}$.

\subsection{Constrained three- and four-point bending tests}

To compare the effect of rubber particles and their content on the plastic deformation and fracture behaviour of the modified epoxies, we used SDEDN specimens tested in four-point bend mode. The geometry of a SDEDN specimen is shown in Fig. 1. The specimen had overall dimensions of $19.1 \mathrm{~mm}$ width $\times 6.3 \mathrm{~mm}$ thickness $\times 126 \mathrm{~mm}$ length, and two notches on each side, which were $25 \mathrm{~mm}$ apart. The notch tip radii were $1.5 \mathrm{~mm}$ for CTB toughened epoxy specimens, and $0.5 \mathrm{~mm}$ for the CTBN toughened epoxy specimens. The $a / W$ ratio was 0.25 , where $a$ is the notch length and $W$ is the width of the specimen. The fourpoint bend spans were 35 and $75 \mathrm{~mm}$ for the upper and lower spans, respectively. The bending crosshead speed was $1 \mathrm{~mm} \mathrm{~min}^{-1}$.

The SDEDN is a further modification of the double-notch four-point bend (DN-4PB) specimen [9], which has two notches both on the tensile side. During testing of a DN-4PB specimen, one of the two identical notches fails, while the other notch is in a sub-critical condition. So, the DN-4PB specimen can provide both fracture and sub-fracture information. The testing of a SDEDN-4PB specimen, however, not only gives us that information, but also allows us to compare the behaviour of rubber particles in tensile and compressive regions.

We also used the sharp-notched three-point bending test to obtain the fracture toughness of the materials. The method was the same as that described by Yee and Pearson [4].

\subsection{Symmetrical double-grooved strip tensile test}

The symmetrical double-grooved strip tensile test has been used by Cost and Parr [10] and Yee et al. [11]. Cost and Parr [10] had theoretically analysed the stress distribution in a strip tensile specimen and found that the specimen's $W / h$ ratio can significantly vary the principal stress ratio, $\sigma_{2} / \sigma_{1}$, i.e. the constraint

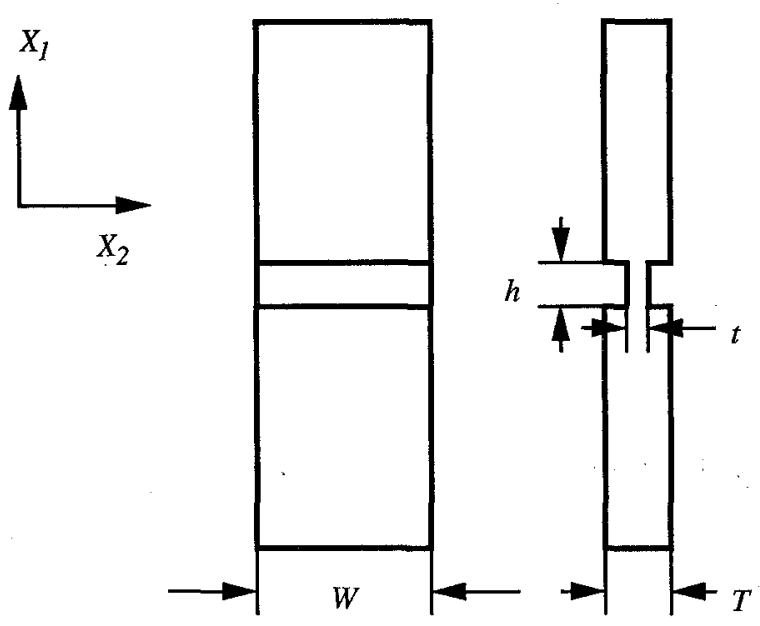

Figure 2 Illustration of a symmetrical double-grooved strip specimen.

in the transverse direction. When the $W / h$ ratio was sufficiently high, a tensile plane strain condition could be created. By using double-grooved specimens, Yee et al. [11] successfully studied the failure of several thermoplastics during their transition from plane stress to plane strain states. We employed this test to study the yield behaviour of rubber-modified epoxies in order to understand the effect of cavitated rubber particles on the constraint created during loading.

The specimens were basically the same as that described elsewhere [11]. However, we made certain changes in the specimen geometry because of the relative brittleness of the epoxy used. The specimen gauge length, $h$, was $3.2 \mathrm{~mm}$, thickness, $t$, was $2.1 \mathrm{~mm}$, width, $w$, varied from $2-25 \mathrm{~mm}$, and the specimen thickness outside the gauge section was $6.3 \mathrm{~mm}$ (Fig. 2). We also machined the grooves using a grinding machine with a fine diamond grinding wheel to reduce the size of surface defects. To ensure that yielding occurred, we used a relatively slow crosshead speed $\left(1 \mathrm{~mm} \mathrm{~min}^{-1}\right)$ in the test.

\subsection{Morphology analysis}

Optical microscopy was used to examine the subsurface damage of fractured specimens, and subcritical damage zones of the constrained four-point bending specimens. In both cases, a thin section perpendicular to the fracture surface or notch surface was obtained by metallographic thinning techniques [12], which included embedding the damage zone portion of a specimen in a clear epoxy resin then reducing the thickness by a combination of cutting and polishing until a final thickness of $25 \mu \mathrm{m}$ or less was reached. The obtained sections were examined under a Nikon research microscope, with or without 
crossed polarizers, for deformations such as birefringence and voiding.

SEM was used to examine the fracture surfaces. In order to reveal subsurface rubber cavitation, SEM was also used to examine microtomed surfaces of the modified epoxies. The instrument used was a Hitachi S-800 scanning electron microscope. Specimens were coated with a thin film of gold by sputtering.

\section{Results and discussion}

\subsection{Constraint relief by rubber cavitation} in SDEDN four-point bending test

\subsubsection{Cracking at the slip-line field boundary in pure epoxy}

When a notched specimen is stressed elastically, it will produce high stresses near the notch tip, and this may locally exceed the material's yield stress to produce a localized plastic zone. For pure polymers, such as epoxy in our study, in both three-point and four-point bending tests, the plastic zone shape can be predicted by the slip-line filed theory $[13,14]$. Because of the strain-softening characteristic of polymers, slip lines can be observed readily. Fig. 3 shows the slip-line pattern in SDEDN epoxy specimen deformed in bending. The slip lines have the appearance of a "rhombohedral" array. However, the angles between the $\alpha$ and $\beta$ lines are found to be approximately $96^{\circ}$, which are in agreement with the modified slip-line field theory for dilative materials [15].

At the plastic-elastic boundary of the slip-line field in pure plastics, the mean stress reaches the highest value. As this maximum increases beyond a critical
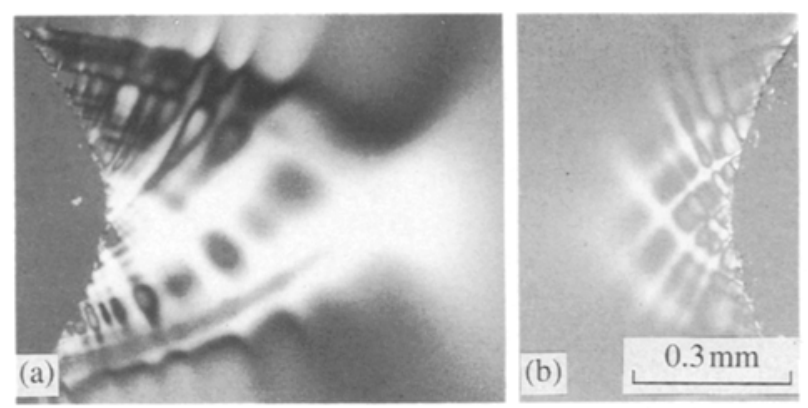

Figure 3 Slip-line pattern in a SDEDN specimen of epoxy: (a) tensile side, (b) compressive side.
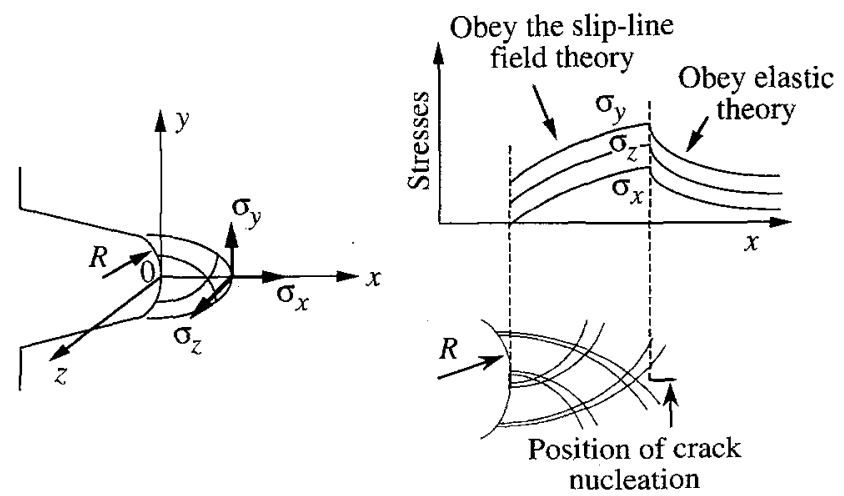

Figure 4 Illustration of the cracking mechanism in a blunt-notched pure epoxy specimen. value, further loading will cause crazing or cracking to occur, which leads to fracture in materials such as PC [14] and epoxy in our study. The development of the slip-line zone and the initiation of a crack at the plastic-elastic boundary of the slip-line zone were observed under a stereomicroscope during the loading of a blunt-notched pure epoxy specimen. The existence of a nucleation site for fracture in the region of the plastic-elastic boundary was further confirmed by SEM fractography (see Fig. 9a). The cracking mechanism in pure epoxy is illustrated in Fig. 4. The highest mean stress at the plastic-elastic boundary is the cause of cracking.

\subsubsection{Effect of CTB rubber on the deformation of modified epoxy}

The CTB rubber, owing to its relatively poor compatibility with the epoxy, formed large particles (diameter as large $100 \mu \mathrm{m}$ ) with a wide size distribution; and the maximum amount of CTB rubber that can be incorporated into our epoxy was about 5 parts per hundred parts of resin (p.h.r.). The CTB rubber was not very effective in increasing the fracture toughness of epoxy because its particles are too large [16]. Despite this fact, by using a relatively blunt notch, the deformation mechanisms were found to be quite similar to those in much tougher systems using small rubber particles. We used the large particles for ease of visualization of the deformation mechanisms because they can be readily observed under an optical microscope.

The sub-critical damage zones of CTB rubbermodified epoxy in both the tensile and the compressive regions are shown in Fig. 5. In the tensile region (Fig. 5a,c), it is obvious that the rubber particles have changed remarkably during the loading process, pre-existing cavities have grown much larger; and new cavities have formed. These cavities exhibit
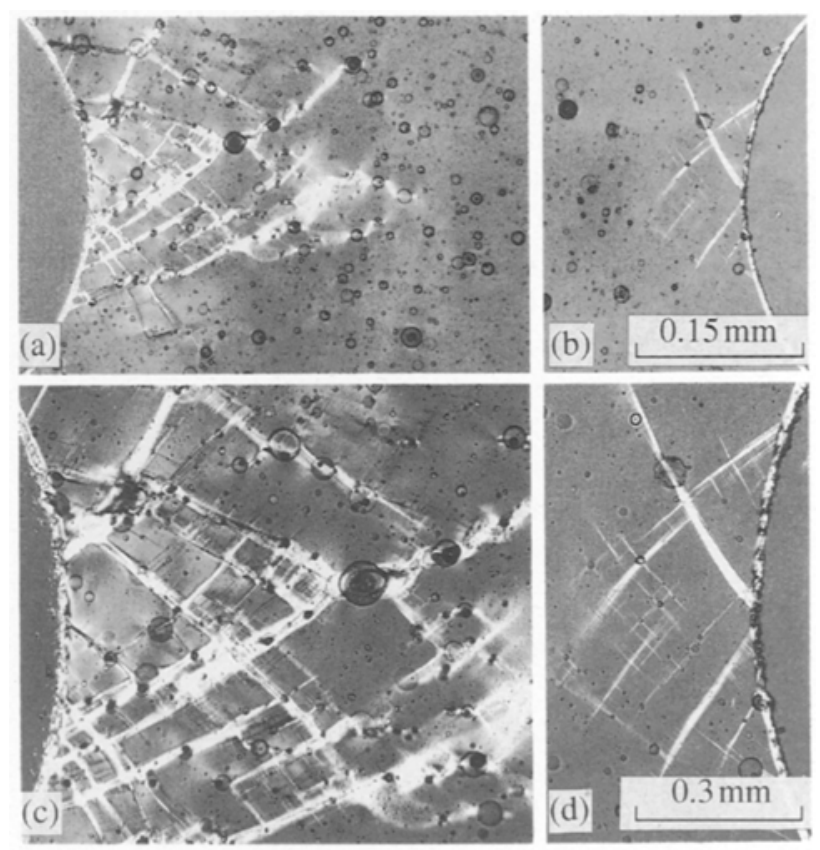

Figure 5 Sub-critical damage zone of 2.5 p.h.r CTB rubber-modified epoxy: $(a, c)$ tensile side, $(b, d)$ compressive side. 

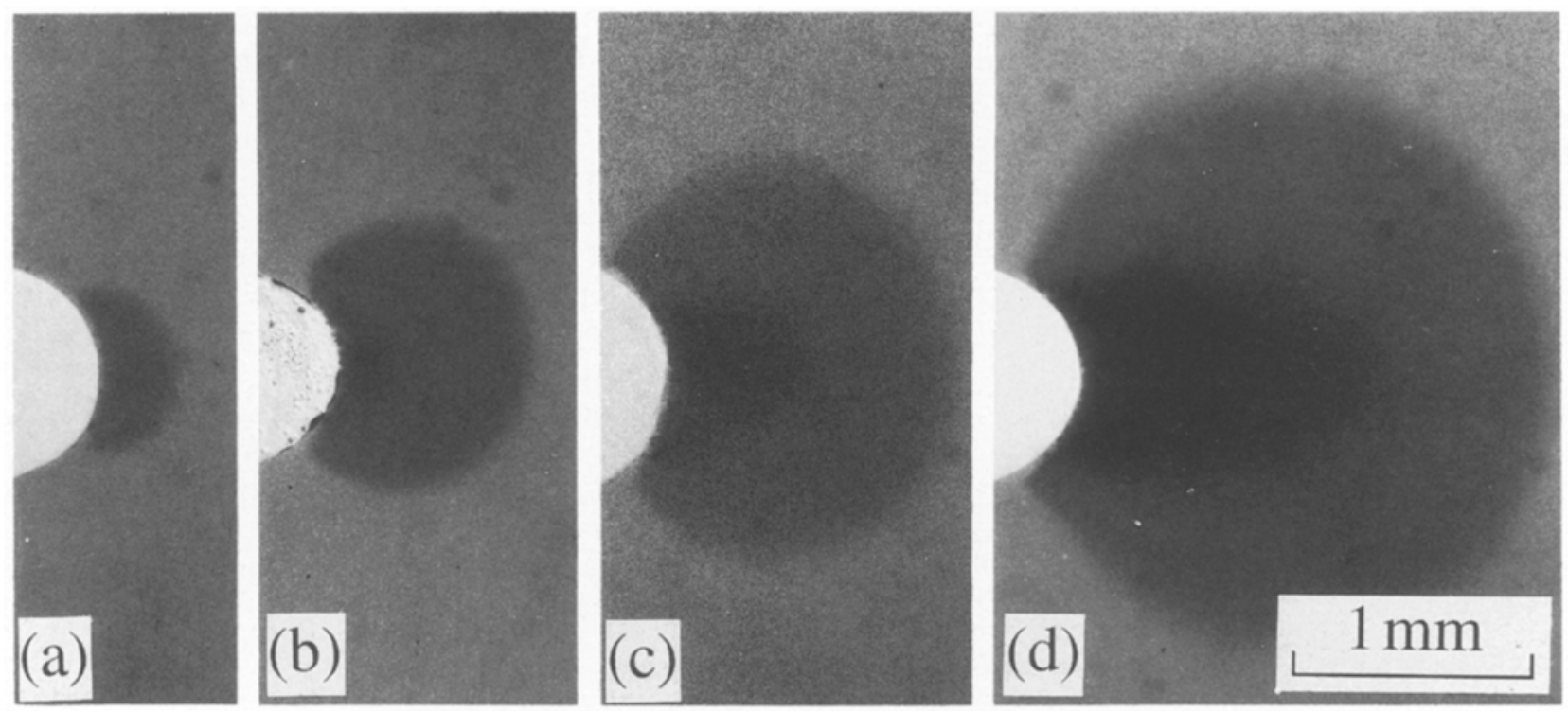

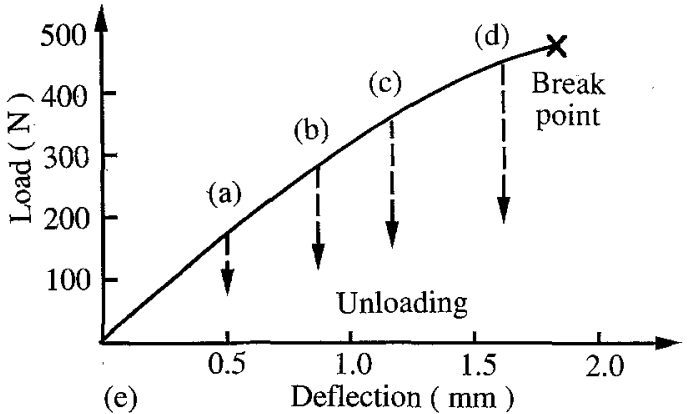

strong interactions with the slip line field, they change the direction of slip lines from the "rhombohedral" array to one which is determined by random encounters with the rubber particles. The directions of some slip lines are modified by such encounters. Some of these particles also initiated secondary slip-line fields. As the rubber cavities grow larger, slip lines also become wider. By contrast, in the compressive region (Fig. 5b,d), pre-existing cavities have disappeared upon loading, and no new cavitation has occurred. No interaction between the rubber particles and the slip lines has been observed. Slip lines encountering rubber particles do not change their directions, and no new slip lines form from such encounters.

Because the rubber particles are substantially softer than the epoxy matrix, they must cause stress concentration when the material is loaded. Yet the above observation indicates that causing stress concentration alone is not enough to induce the matrix to undergo shear yielding; the totally different effects of rubber particles on the plastic zone demonstrate the necessity of cavitation of the rubber particles.

\subsubsection{Effect of CTBN rubber on the deformation of modified epoxy}

The effect of a different CTBN rubber with $18 \%$ acrylonitrile as a copolymer content was also studied. Because of the greater solubility, this rubber forms relatively fine particles (diameter about $2 \mu \mathrm{m}$ ) with narrow size distribution in the epoxy. As much as 30 p.h.r. of this CTBN rubber can be added to the
Figure 6 (a-d) The development of cavitation and plastic zone of 10 p.h.r. CTBN rubber-modified epoxy in blunt-notched bending test. The level of loading for each specimen is indicated in the load-deflection curve (e).

epoxy. As a result of these differences, the fracture toughness of this CTBN rubber-modified epoxy is several times higher than that of pure epoxy.

In a deformed SDEDN specimen of CTBN rubbermodified epoxy, a slip-line field can also be seen at the notch tip, and rubber cavitation is observed together with the plastic (slip-line) zone. By using five specimens of identical geometry, but loading them to various levels of deflection prior to fracture, and then unloading them and making thin sections as introduced before, we were able to observe the development of cavitation and plastic zones in 10 p.h.r. CTBN rubber-modified epoxy (Fig. 6). The cavitation and plastic zones both grow with increased loading. At a very small load, the rubber cavitation has already occurred (Fig. 6a), and at a certain later stage the plastic zone begins to occur (Fig. 6b). A scanning electron micrograph of a microtomed surface shows that in the plastic zone, rubber cavitation is very obvious (Fig. 7). The above observation shows that rubber cavitation precedes plastic yielding. We note that the amount by which cavitation precedes plastic yielding must depend on the cavitation resistance of the rubber. At room temperature and at moderate strain rates this particular CTBN appears to cavitate well before shear yielding in the matrix occurs. However, some other types of rubber tougheners, e.g. MBS-type core-shell particles cavitate just before matrix shear yielding occurs.

We then investigated the effect of rubber content on the deformation and fracture behaviour of the modified materials. We tested SDEDN specimens of CTBN rubber-modified epoxies in four-point bend. The subcritical damage zones of 2.5-13.5 p.h.r. CTBN rubbermodified epoxy specimens were compared with that of a pure epoxy specimen, Fig. 8. In the compressive regions, the sizes of the plastic zones are nearly identical even though the rubber contents increase from 0 to 13.5 p.h.r. By contrast, in the tensile regions, the 


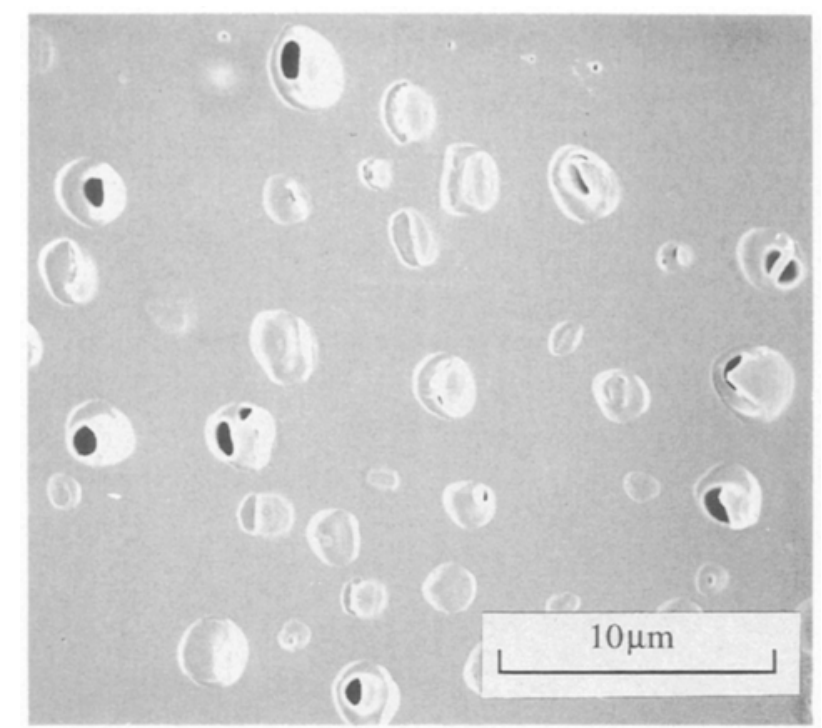

Figure 7 Cavitation in the plastic zone as revealed in a scanning electron micrograph of a microtomed surface.

plastic zones increase systematically as the rubber content increases. SEM images of the microtomed surface again confirmed that in the tensile plastic zone, rubber cavitation has occurred, whereas in the compressive plastic zone, no rubber cavitation was evident.

Another important feature observed in these specimens (Fig. 8) was that the cavitation zone decreased as the rubber content increased. We know that rubber cavitation occurs at a certain critical mean stress/ strain. The decreasing cavitation zone size indicates that the mean stress ahead of the notch tip decreases with increasing rubber content. One reasonable explanation for the mean stress reduction is the constraint relief effect by cavitation inside the rubber particles. The greater the number of cavitated rubber particles, the greater is this constraint relief effect. So, the mean stress in materials with lower rubber concentrations can reach higher values and spread over wider regions. For the same reason, the mean stress in materials with higher rubber concentrations is lower and spreads over smaller regions. Because reaching the critical mean stress is a necessary condition for cracking in our epoxy, by adding more and more rubber, we can lower the mean stress, postpone the cracking process, and thus allow more plastic deformation to occur. This is the reason why materials with higher rubber contents have larger plastic zones, but smaller cavitation zones.

In summary, these observations reaffirm that without rubber cavitation, even though the rubber particles could still cause stress concentration, additional shear deformation is not induced. With cavitation, significantly more plastic deformation can occur in the modified materials.

\subsubsection{Effect of CTBN rubber on the fracture mode of modified epoxy}

SEM fractography shows that there is even a fracture mode change as the rubber content increases. The fracture surface of a blunt notched pure epoxy specimen indicates that a crack initiation site existed ahead of the notch tip (Fig. 9a). This site corresponds to the boundary of the plastic-elastic zone [14]. The fracture surface of 2.5 p.h.r. CTBN rubber-modified epoxy also exhibited such a crack initiation site ahead of the notch tip (Fig. 9b). While an initiation site also existed in 7.5 p.h.r. CTBN rubber-modified epoxy, its location was significantly closer to the notch tip (Fig. 9c). Finally, for 10 p.h.r. or higher CTBN rubber-modified epoxies, the nucleation site was located right at the notch tip (Fig. 9d). This evidence could mean that the elastic-plastic boundary, where the mean stress is a maximum (Fig. 4), is closer to the notch tip as the rubber concentrations increases. Alternatively, this effect could be due to a change of the fracture mode from plane strain to plane stress. In the latter scenario, the fracture mode in plane strain would be controlled by cracking due to the highest mean stress, while the
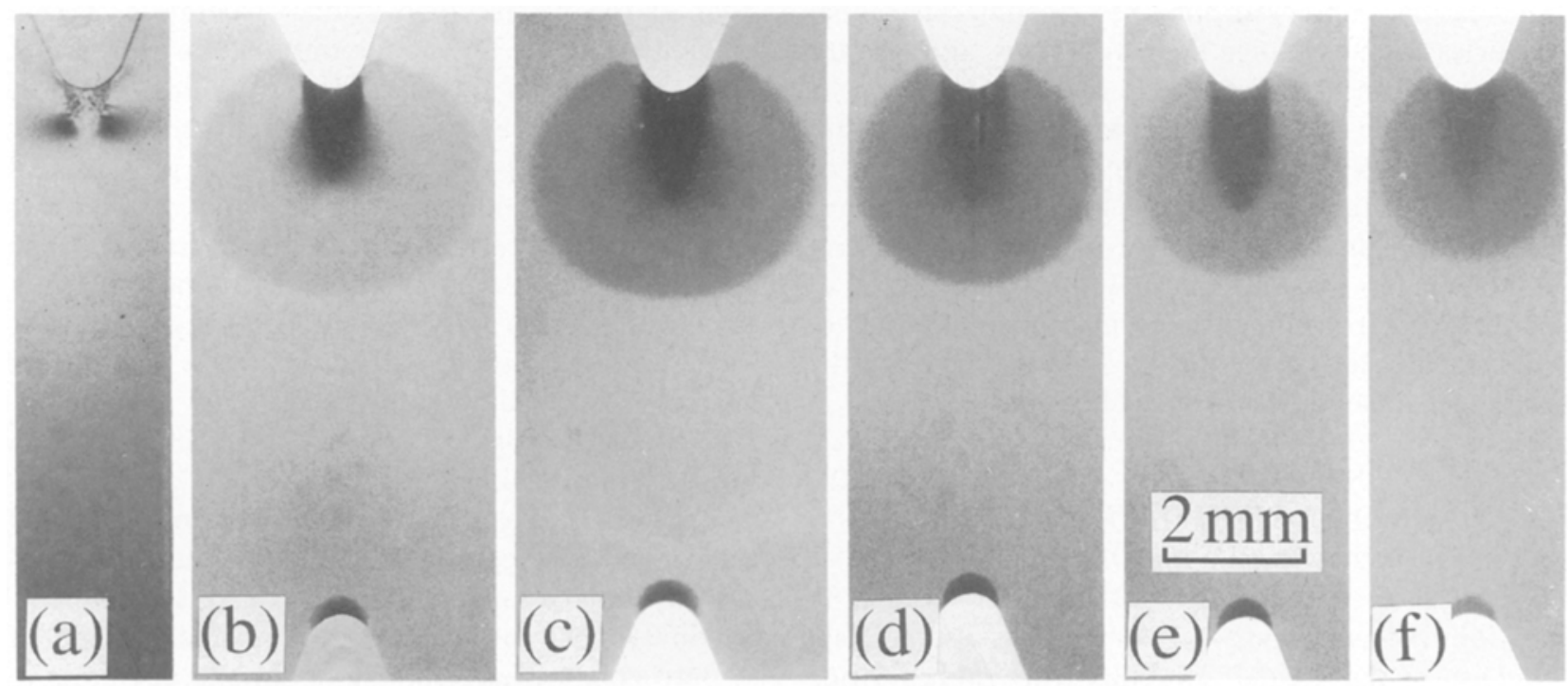

Figure 8 Sub-critical damage zones of SDEDN four-point bend specimens of (a) pure epoxy, (b) 2.5 p.h.r. (c) 5.0 p.h.r. (d) p.h.r. (e) 10.0 p.h.r. (f) 13.5 p.h.r. CTBN rubber-modified epoxies. Note that the notches at the top of the micrographs are in tension, whereas those at the bottom of the micrographs are in compression. 

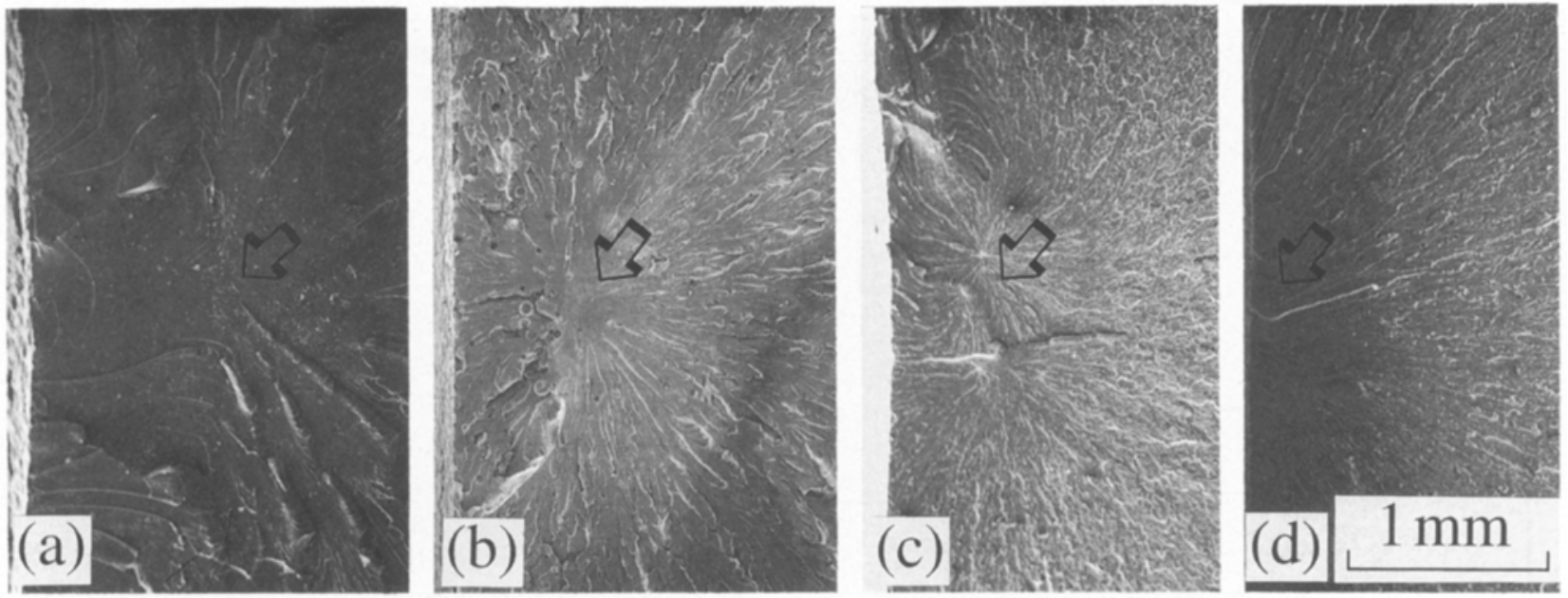

Figure 9 Scanning electron micrographs showing the nucleation sites of cracks in blunt-notched pure and rubber-modified epoxy specimens. (a) Pure epoxy, (b) 2.5 p.h.r., (c) 7.5 p.h.r., (d) 10.0 p.h.r. CTBN rubber-modified epoxies.

fracture mode in plane stress would be controlled by the ultimate tensile strain of the material at the notch tip because local strain is the highest there. Because the plastic zone size increases monotonically with rubber concentration, it is unlikely that the location of the maximum mean stress approaches the notch tip with increasing rubber concentration if the fracture mode remains in plane strain. Consequently, there must have been a mode change from plane strain to plane stress. This change is brought about by the constraint relief effect as shown in the next section.

It is well known in fracture mechanics that the plastic zone at a crack tip is larger by a factor of two to three if the stress is plane rather than plane strain [17]. The experimental results here are consistent with this prediction in a qualitative sense, although the absence of a sharp crack prevents us from making a quantitative comparison with this prediction.

\subsection{The effect of constraint relief by rubber cavitation in the strip tensile test}

The symmetrical double-grooved strip tensile test (hereafter referred to simply as the strip tensile test) is employed to justify further the explanations given in the previous section. In the strip tensile test, an increasing transverse constraint is created by increasing the $W / h$ ratio of the specimens (Fig. 2), which will eventually induce a tensile plant condition. As a result of this transverse constraint, the apparent yield stress of a polymer obeying a von Mises-type yield criterion will increase as the $W / h$ value increases (Fig. 10), reflecting the difference between plane stress and plane strain states $[10,11]$.

For the pure epoxy, the apparent yield stress in the tensile direction increased as the $W / h$ ratio increased (Fig. 11), indicating that a higher constraint was indeed created in specimens with larger $W / h$ ratio. Further, at a $W / h$ ratio of 3 or higher, the specimens failed by cracking prior to achieving yield. This is consistent with our previous results which show that the pure epoxy failed by cracking at the critical mean stress.

Unlike the pure epoxy, the apparent yield stress of 10 p.h.r. rubber-modified epoxy does not increase with

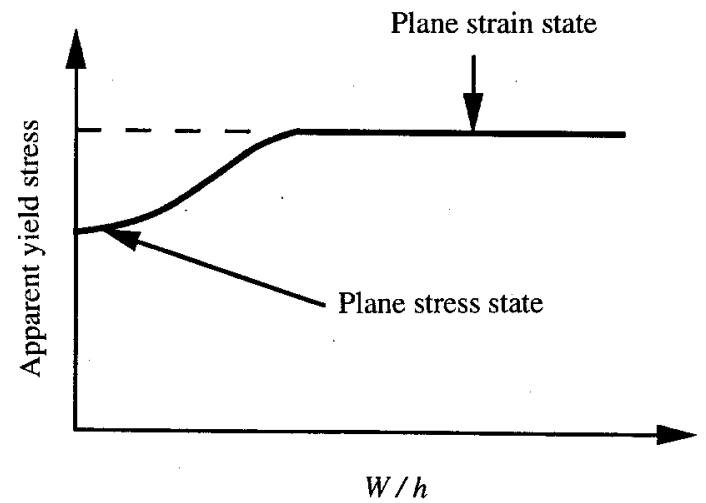

Figure 10 Schematic illustration of the apparent yield stress versus $W / h$ for a von Mises-type polymer.

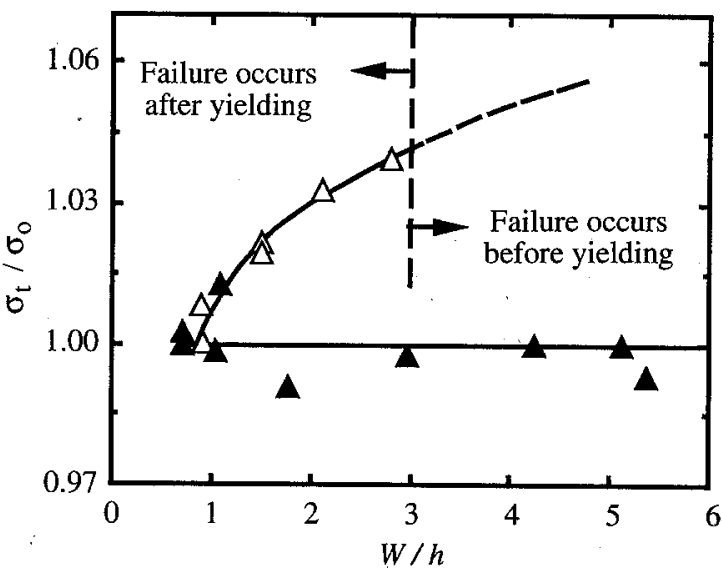

Figure 11 Normalized apparent yield stress in tensile direction $\sigma_{\sqrt{ }} / \sigma_{\mathrm{O}}$, of pure and rubber-modified epoxies versus $W / h$ ratio. $(\triangle)$ Pure epoxy, $(\triangle) 10.0$ p.h.r. CTBN rubber-modified epoxy.

$W / h$ ratio at all (Fig. 11). Thus there is no increase in constraint with specimen width. Further, all specimens tested with 10 p.h.r. rubber failed after shear yielding had occurred, and no brittle failure was observed. SEM images of microtomed surfaces show that rubber cavitation had occurred in the gauge section.

Following the same argument as in the previous section, these results can be explained by the following sequence of microscopic events: (a) rubber particles 


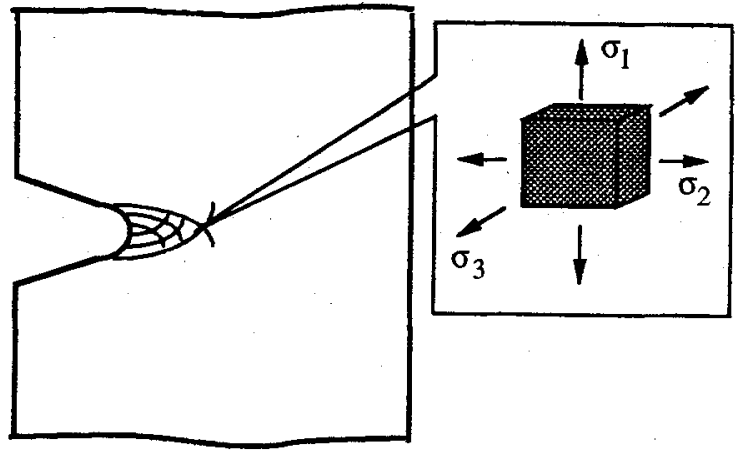

(a)

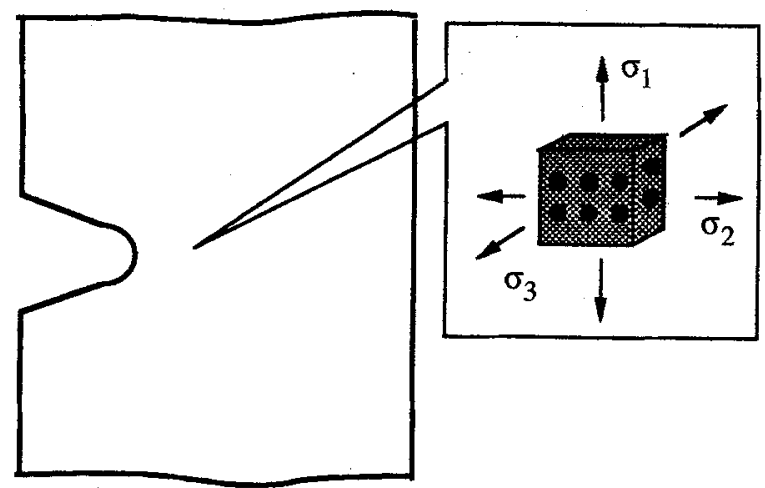

(b)

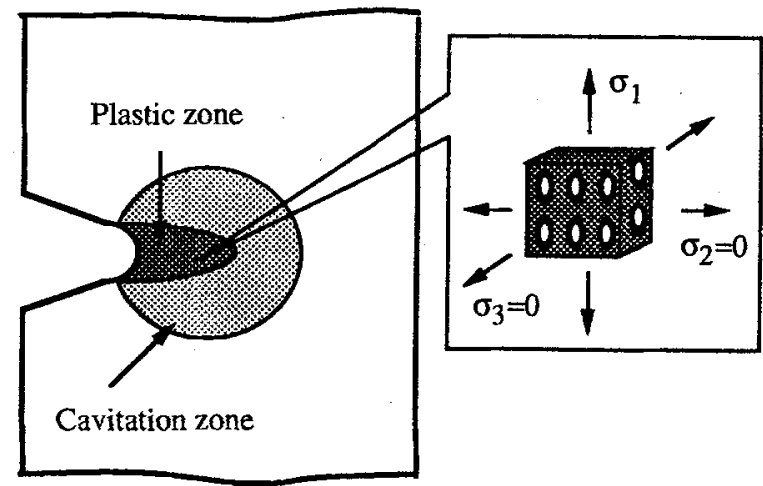

(c)

Figure 12 Illustration of the deformation mechanisms in bluntnotched pure and CTBN rubber-modified epoxy specimens. (a) Pure epoxy: triaxial tensile stresses build up in front of the notch in the bending test. (b) Rubber-modified epoxy: before rubber particle cavitation, it behaves like pure epoxy. (c) Rubber-modified epoxy: after rubber particle cavitation, the constraints in the transverse directions are relieved, the matrix epoxy deforms in the plane stress state if the rubber concentration is sufficiently high.

cavitate after a critical mean stress value is reached, (b) these cavitated rubber particles can no longer support load; more specifically, the surfaces of the cavities can no longer support triaxial tension, hence constraint relief is effected within the material, (c) with a sufficiently larger number of cavities, constraint relief causes the stress state to change towards a plane stress state. Although a thick specimen is used to create a plane strain condition, the plane strain state cannot be reached. As a result of this, the matrix epoxy can deform without constraint, which is more favourable for shear yielding. The deformation mechanisms in rubber-modified epoxies based on the above analyses are illustrated in Fig. 12.

\section{Conclusion}

It can be concluded that the success of CTBN rubber as a toughening agent for epoxies is closely related to its ability to cavitate. The rubber cavitation causes local constraint relief in the material. Given sufficient rubber cavitation, the stress state in a constrained body can be changed to a plane stress state. As a result of this constraint relief, the crack initiation process in the epoxy matrix is circumvented, and the surrounding epoxy matrix can undergo more plastic deformation and absorb more plastic energy.

\section{Acknowledgement}

This work was supported by a MRG Grant from the NSF, DMR 8708405.

\section{References}

1. A. C. GARG and Y.-W. MAI, Compos. Sci. Technol. 31 (1988) 179.

2. W. D. BASCOM and D. L. HUNSTON, in "Adhesion 6", edited by K. W. Allen (Applied Science, London, 1980).

3. D. L. HUNSTON and W. D. BASCOM, in "Advances in Chemistry Series", No. 208, "Rubber Modified Thermoset Resins", edited by C. K. Riew and J. K. Gillham (American Chemical Society, 1984) p. 83.

4. A. F. YEE and R. A. PEARSON, J, Mater. Sci. 21 (1986) 2462.

5. R. A. PEARSON and A. F. YEE, ibid. 21 (1986) 2475.

6. A. J. KINLOCH, S. J. SHAW, D. A. TOD and D. L. HUNSTON, Polymer 24 (1983) 1341.

7. A. J. KINLOCH, S. J. SHAW and D. L. HUNSTON, ibid. 24 (1983) 1356

8. C. K. RIEW, E. H. ROWE and A. R. SIEBERT, $A C S A d v$. Chem. Ser. 154 (1976) 326.

9. H.-J. SUE and A. F. YEE, J. Mater. Sci. 24 (1989) 1447.

10. T. L. CosT and C. H. PAR R, J. Mater. 4 (1969) 312.

11. A. F. YEE, W. V. OLSZEWSKI and S. MILLER, in "Advances in Chemistry Series", No. 154, edited by R. D. Deanin and A. M. Crugnola (American Chemical Society, 1976) p. 97.

12. A. S. HOLIK, R. P. KAMPOUR, S. Y. HOBBS and D. G. FINK, Microstruct. Sci. 7 (1979) 357

13. R. HILL, in "The Mathematical Theory of Plasticity" (Oxford University Press, 1950).

14. M. ISHIKAWA, I. NARISAWA and H. OGAWA $J$. Polym. Sci. 15 (1977) 1791.

15. F. Z. LI and J. PAN, J. Appl. Mech. Trans. ASME 57 (1990) 259.

16. R. A. PEARSON and A. F. YEE, J. Mater. Sci. 26(1991) 3828.

17. D. BROEK, in "Elementary Engineering Fracture Mechanics" (Martinus Nijhoff, Boston) p. 107.

Received 20 July 1992

and accepted 27 April 1993 